\title{
Prevalence and goal attainment with spinal orthoses for children with cerebral palsy
}

\author{
Katina Pettersson ${ }^{\mathrm{a}, \mathrm{b}, *}$ and Elisabet Rodby-Bousquet ${ }^{\mathrm{a}, \mathrm{b}}$ \\ ${ }^{a}$ Department of Clinical Sciences Lund, Lund University, Orthopaedics, Lund, Sweden \\ ${ }^{\mathrm{b}}$ Centre for Clinical Research Västerås, Uppsala University, Västerås, Sweden
}

\begin{abstract}
.
PURPOSE: Analyze the goals for treatment and attained goals for spinal orthoses in children with cerebral palsy (CP), and describe the use of spinal orthoses in relation to age, sex, gross motor function, and scoliosis.

METHODS: Cross-sectional data for all children born between 2000 and 2014 and registered in the Swedish CP registry were analyzed in relation to age, sex, Gross Motor Function Classification System (GMFCS), and scoliosis. Treatment goals were to 1) prevent deformity; 2) improve stability/positioning; 3) improve head control; and 4) improve arm/hand function.

RESULTS: Overall, 251 of the 2800 children (9\%) used spinal orthoses, and the frequency increased significantly with age and GMFCS level; 147 of the 251 children had scoliosis. Several treatment goals were reported for most children. The most common goal was improved stability/positioning (96\%), followed by head control (51\%) and arm/hand function (38\%). Only one third of the children used spinal orthoses to prevent deformities. The rate of goal attainment was $78-87 \%$ for the functional outcomes and $57 \%$ for the prevention of deformities.

CONCLUSION: Although the goal of using spinal orthosis to prevent curvature progression remains important, we found that its functional benefits (stability, head control, arm/hand function) were of greater importance.
\end{abstract}

Keywords: Cerebral palsy, children, adolescents, spinal orthoses, goals, postural balance

\section{Introduction}

\subsection{Cerebral palsy}

Movement and posture are key problems for children with cerebral palsy (CP) [1]. These may present as difficulties with the adaptation and fine tuning of postural muscles, which possibly cause impaired stability [2]. A lack of stability can affect both head and hand control [3]. Without adequate support, there is an increased risk that children will develop functional quadriplegia and require higher levels of care and nursing [4], thereby reducing the individual's independence. The severity of motor impairment can be classified according to the Gross Motor Function Clas-

${ }^{*}$ Corresponding author: Katina Pettersson, Department of Clinical Sciences Lund, Orthopaedics, Lund University, SE-221 85 Lund, Sweden. Tel.: +46 2117 5887; E-mail: katina.pettersson@med.lu.se. sification System (GMFCS) [5]. Inability to deal with the effects of gravity, combined with immobility, spasticity, and muscle weakness, lead to an increased risk of developing muscle contractures, skeletal deformities, hip dislocation, and scoliosis from early childhood [6].

\subsection{Spinal orthoses}

Lack of stability is often the main indication for supports such as spinal orthoses [7,8]. Previous studies have reported that spinal orthoses can provide functional benefits such as stability [9], head control [7,10], and hand control $[7,8]$, all of which preserve and improve functional abilities that are vital to the quality of life for children with CP. Spinal orthoses appear to be effective for the treatment of adolescent idiopathic scoliosis [11]; however, the results for neuromuscular scoliosis are more ambiguous $[8,9,12]$. The main 
indication for spinal orthoses in these patients is often to stabilize and delay the progression of the spinal curvature [8,12]. Nevertheless, there remains a lack of knowledge regarding the treatment goals and the level of goal attainment for the use of spinal orthoses in children with CP [6].

\subsection{Scoliosis}

Posture and stability can also be affected by scoliosis $[7,13,14]$. Scoliosis develops in approximately $25 \%$ of children with CP, and can be a significant problem depending on the child's age, neurological subtype, and GMFCS level [14]. Scoliosis may progress into adulthood with a higher risk for individuals at GMFCS levels IV and V [15]. Pronounced scoliosis not only affects the spine but also has a great impact on quality of life and factors such as sitting ability, transfers, pain, and care needs [16]. Scoliosis that is left untreated can cause respiratory and cardiac complications and premature death [4]. Other available approaches that aim to improve stability include adaptive seating, orthotics, and postural management [13,17], while surgery may be the definitive treatment option for scoliosis $[9,13,17]$.

\subsection{Aim}

Currently, there is a lack of knowledge regarding how spinal orthoses are used in the population of children with CP. The aim of the present study was to analyze the treatment goals for, and goal attainment levels with, spinal orthoses in a population of children with $\mathrm{CP}$ and to describe the use of spinal orthoses in relation to age, sex, gross motor function, and scoliosis.

\section{Material and methods}

\subsection{Ethical approval and consent}

The study was approved by the Medical Research Ethics Committee at Lund University, and permission was obtained to extract data from the Swedish National Cerebral Palsy Surveillance Program and Registry (CPUP). All participants consented to contribute to research based on reported data.

\subsection{Data collection and participants}

This cross-sectional study included all children with CP in Sweden born between 2000 and 2014 who were reported to and included in CPUP. Data were extracted from the most recent physiotherapy assessment for each child between 1 January 2013 and 31 December 2014. CPUP was initiated in the south of Sweden in 1994 to prevent hip dislocations, contractures, and deformities in children with CP $[18,19]$. This combined surveillance program and registry represents all children with CP in Sweden with a coverage rate of over 95\% [20]. The cohort includes children aged younger than 4 years with suspected but not yet confirmed CP. Usually, the child's diagnosis is verified by a neuropediatrician at the age of 4 years, and children who do not fulfil the criteria are excluded. Exclusion and inclusion criteria are in accordance with those of the Surveillance of Cerebral Palsy network in Europe, including a brain injury occurring before the age of 2 years [21].

\subsection{Classifications and measurements}

The children were examined twice a year by their local physiotherapist according to a standardized protocol until the age of 6 years and once a year thereafter [22]. The measurements were reported on a web-based survey form and are stored in the CPUP database.

\subsubsection{Gross Motor Function Classification System}

Each child's gross motor skills were classified according to the expanded and revised version of the GMFCS with the additional age band for 12 to 18 years, where level I describes the highest and level $\mathrm{V}$ describes the lowest functional level [5].

\subsubsection{Clinical examination of the spine}

A clinical examination of the spine was performed by the physiotherapist with the child in a sitting position on a plinth, both in a forward bend and upright. This spinal examination has been evaluated for psychometric properties and shows a high concurrent validity compared with the radiographic Cobb angle, having a sensitivity of $75 \%$ (95\% confidence interval [CI]; 19.4-99.4), specificity of 95.8\% (95\% CI; 78.9-99.9), and excellent interrater reliability (kappa $=0.96$ ) [23]. Curvature of the spine was graded as follows.

- No Scoliosis.

- Mild: A discreet curvature visible only on a thorough examination during forward bending.

- Moderate: An obvious curvature visible during both upright and forward bending positions.

- Severe: A pronounced curvature preventing the child from attaining an upright position without external support. 
Table 1

Details of the 2800 participants aged $0-14$ years

\begin{tabular}{|c|c|c|c|c|c|c|c|c|}
\hline \multirow[t]{2}{*}{ Age } & \multirow{2}{*}{$\begin{array}{c}\text { Number of } \\
\text { children }\end{array}$} & \multicolumn{2}{|c|}{ Sex } & \multicolumn{5}{|c|}{ GMFCS } \\
\hline & & Boys & Girls & I & II & III & IV & $\mathrm{V}$ \\
\hline$<1$ & 6 & 3 & 3 & 0 & 3 & 0 & 0 & 3 \\
\hline 1 & 91 & 55 & 36 & 29 & 21 & 9 & 17 & 15 \\
\hline 2 & 170 & 95 & 75 & 74 & 22 & 22 & 26 & 26 \\
\hline 3 & 202 & 111 & 91 & 88 & 30 & 25 & 27 & 32 \\
\hline 4 & 215 & 121 & 94 & 99 & 34 & 19 & 36 & 27 \\
\hline 5 & 295 & 178 & 117 & 144 & 37 & 28 & 48 & 38 \\
\hline 6 & 246 & 142 & 104 & 118 & 35 & 26 & 28 & 39 \\
\hline 7 & 233 & 138 & 95 & 100 & 48 & 19 & 24 & 42 \\
\hline 8 & 237 & 146 & 91 & 97 & 42 & 23 & 44 & 31 \\
\hline 9 & 194 & 101 & 93 & 80 & 31 & 16 & 34 & 33 \\
\hline 10 & 233 & 148 & 85 & 121 & 36 & 13 & 31 & 32 \\
\hline 11 & 206 & 115 & 91 & 76 & 38 & 15 & 32 & 45 \\
\hline 12 & 217 & 119 & 98 & 91 & 40 & 19 & 33 & 34 \\
\hline 13 & 168 & 87 & 81 & 57 & 40 & 11 & 31 & 29 \\
\hline 14 & 87 & 55 & 32 & 36 & 13 & 9 & 12 & 17 \\
\hline Total & 2800 & 1614 & 1186 & 1210 & 470 & 254 & 423 & 443 \\
\hline
\end{tabular}

\subsection{Spinal orthoses - treatment goals and goal attainment}

The use of a spinal orthosis was reported as "yes" or "no" for each child. All prefabricated or individually molded spinal orthoses were included. Treatment goals were set for each child by collaboration between the family and therapist based on four possible options available in the database: 1) prevent deformity, 2) improve stability/positioning, 3) improve arm/hand function, and 4) improve head control. Note that several goals could be reported for each child, depending on the child's individual needs. In this study, we considered goal 1 to focus mainly on body structure, and the prevention of deformity was defined as prevention, reduction, or stabilization of scoliosis. Goals 2, 3, and 4 focus more on activities and body function, and are hereafter referred to as functional goals. Attainment for each goal chosen was noted as "yes" or "no", that is, whether or not the child attained the intended goal using the spinal orthosis. All responses were reported by the physiotherapists in cooperation with the children and their families.

\subsection{Statistical analyses}

For descriptive statistics, categorical data were reported as frequencies and percentages, $n(\%)$, while discrete and continuous data were reported as medians, means, and standard deviations (SD). Nonparametric tests including chi-square and chi-square for trends were used to analyze the differences between variables. We considered $p$ values $<0.05$ to be significant. IBM SPSS Statistics v24.0 (IBM Statistics, Armonk NY), was used for all statistical analyses.

\section{Results}

\subsection{Demographics}

In total, results from 2800 children ( $58 \%$ boys, $42 \%$ girls) with a median age of 7 years (range $0-14$ years) were reported to the CPUP registry. The children were classified at GMFCS levels I (43\%), II (17\%), III $(9.1 \%)$, IV $(15 \%)$, and V $(16 \%)$. The distributions of age, sex, and GMFCS levels are presented in Table 1.

\subsection{Spinal orthoses}

Overall, $251(9 \%)$ of the 2800 children, 135 (54\%) boys and $116(46 \%)$ girls, used spinal orthoses. Their median age was 8.0 years (range 1-14 years). The proportion of children using spinal orthoses increased with age $(p<0.001)$, from less than $4 \%$ in 2 -year-olds up to $13 \%$ in 9 -year-olds. Spinal orthoses were only used by children at GMFCS levels III to V and use was more frequent in children at lower levels of motor function $(p<0.001)$, ranging from less than $2 \%$ at GMFCS level III to $38 \%$ of all children classified at GMFCS level V (Table 2). Of the 251 children who used spinal orthoses, 147 (59\%) had scoliosis, representing 30\% of all children with scoliosis (see Section 3.3 Scoliosis). Information regarding the presence or absence of scoliosis was missing for two children. The scoliosis was reported as mild in 56, moderate in 39 , and severe in 43 children. Information regarding the severity of the scoliosis was missing for nine children. The remaining 102 children (41\%) did not have scoliosis but used spinal orthoses either to improve function or to prevent deformity (Table 2). 
Table 2

The use of spinal orthoses in numbers and percentages of children with cerebral palsy, the structural and functional treatment goals and the rate of goal attainment relative to their age, sex, GMFCS level and degree of scoliosis

\begin{tabular}{|c|c|c|c|c|c|c|c|c|c|c|}
\hline \multirow[b]{2}{*}{ Age } & \multirow{2}{*}{$\begin{array}{c}\text { Total } \\
\text { number } \\
\mathrm{N}\end{array}$} & \multirow{2}{*}{$\begin{array}{c}\text { Spinal } \\
\text { orthosis } \\
\mathrm{N}(\%)\end{array}$} & \multicolumn{2}{|c|}{$\begin{array}{c}\text { Prevent } \\
\text { deformity }\end{array}$} & \multicolumn{2}{|c|}{$\begin{array}{c}\text { Improve } \\
\text { stability/positioning }\end{array}$} & \multicolumn{2}{|c|}{$\begin{array}{l}\text { Improve arm/hand } \\
\text { function }\end{array}$} & \multicolumn{2}{|c|}{$\begin{array}{l}\text { Improve head } \\
\text { control }\end{array}$} \\
\hline & & & $\mathrm{G}$ & GA (\%) & $\mathrm{G}$ & GA (\%) & $\mathrm{G}$ & GA (\%) & $\mathrm{G}$ & GA $(\%)$ \\
\hline$<1$ & 6 & $0(0.0)$ & 0 & $0(0.0)$ & 0 & $0(0.0)$ & 0 & $0(0.0)$ & 0 & $0(0.0)$ \\
\hline 1 & 91 & $1(1.1)$ & 1 & $0(0.0)$ & 1 & $0(0.0)$ & 0 & $0(0.0)$ & 0 & $0(0.0)$ \\
\hline 2 & 170 & $6(3.5)$ & 1 & $0(0.0)$ & 5 & $5(100)$ & 3 & $2(67)$ & 5 & $4(80)$ \\
\hline 3 & 202 & $14(6.9)$ & 2 & $1(50)$ & 14 & $12(86)$ & 5 & $4(80)$ & 7 & $6(86)$ \\
\hline 4 & 215 & $14(6.5)$ & 4 & $2(50)$ & 13 & $11(85)$ & 8 & $6(75)$ & 10 & $6(60)$ \\
\hline 5 & 295 & $27(9.2)$ & 5 & $4(80)$ & 25 & $24(96)$ & 12 & $11(92)$ & 15 & $12(80)$ \\
\hline 6 & 246 & $19(7.7)$ & 10 & $7(70)$ & 19 & $16(84)$ & 8 & 7 (88) & 12 & $10(83)$ \\
\hline 7 & 233 & $2(10)$ & 8 & $6(75)$ & 23 & $20(87)$ & 9 & $7(78)$ & 12 & 7 (58) \\
\hline 8 & 237 & $25(10)$ & 7 & $4(57)$ & 24 & $21(88)$ & 11 & $8(73)$ & 13 & $11(85)$ \\
\hline 9 & 194 & $26(13)$ & 7 & $5(71)$ & 26 & $22(85)$ & 8 & $7(88)$ & 10 & $9(90)$ \\
\hline 10 & 233 & $19(8.2)$ & 8 & $7(88)$ & 18 & 17 (94) & 5 & $5(100)$ & 10 & $8(80)$ \\
\hline 11 & 206 & $25(12)$ & 9 & $4(44)$ & 24 & 19 (79) & 5 & $4(80)$ & 10 & $8(80)$ \\
\hline 12 & 217 & $24(11)$ & 11 & $4(36)$ & 24 & $21(88)$ & 12 & $9(75)$ & 12 & $11(92)$ \\
\hline 13 & 168 & $19(11)$ & 5 & $2(40)$ & 18 & $16(89)$ & 6 & $5(83)$ & 7 & $4(57)$ \\
\hline 14 & 87 & $8(9.2)$ & 5 & $1(20)$ & 7 & $5(71)$ & 4 & $2(50)$ & 4 & $3(75)$ \\
\hline \multicolumn{11}{|l|}{ Sex } \\
\hline Boys & 1614 & $135(8.4)$ & 44 & $22(50)$ & 132 & $115(87)$ & 50 & $40(80)$ & 72 & $57(72)$ \\
\hline Girls & 1186 & $116(9.8)$ & 39 & $25(64)$ & 109 & 94 (86) & 46 & $37(80)$ & 55 & $42(76)$ \\
\hline \multicolumn{11}{|l|}{ GMFCS } \\
\hline III & 254 & 4 (1.6) & 1 & $0(0.0)$ & 3 & $2(67)$ & 1 & $1(100)$ & 0 & $0(0.0)$ \\
\hline IV & 423 & 77 (18) & 23 & $14(61)$ & 76 & $68(89)$ & 51 & $40(78)$ & 38 & $31(82)$ \\
\hline $\mathrm{V}$ & 443 & $170(38)$ & 59 & $33(56)$ & 162 & $139(80)$ & 44 & $36(81)$ & 89 & $68(76)$ \\
\hline \multicolumn{11}{|l|}{ Scoliosis } \\
\hline No & 2312 & $113(4.8)$ & 26 & $18(69)$ & 110 & $97(88)$ & 54 & $44(81)$ & 68 & $54(79)$ \\
\hline Mild & 333 & $56(17)$ & 14 & $10(71)$ & 55 & $44(80)$ & 24 & $20(83)$ & 25 & $18(72)$ \\
\hline Moderate & 71 & $39(55)$ & 16 & $9(56)$ & 36 & 34 (94) & 5 & $4(80)$ & 14 & $10(71)$ \\
\hline Severe & 64 & $43(67)$ & 27 & $10(37)$ & 40 & $34(85)$ & 13 & $9(69)$ & 20 & $17(85)$ \\
\hline Total & 2800 & 251 & 83 & 47 (57) & 241 & 209 (87) & 96 & 77 (80) & 127 & $99(78)$ \\
\hline
\end{tabular}

GMFCS = Gross Motor Function Classification System; $\mathrm{N}=$ number of children per goal; $\mathrm{G}=$ goal; GA = goal attainment .

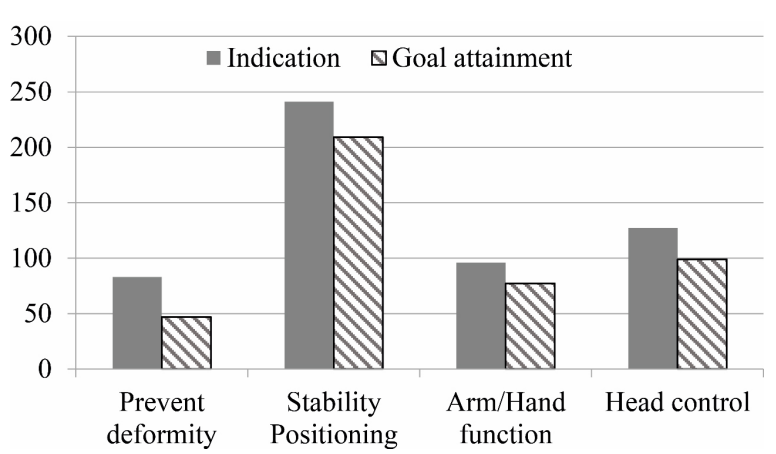

Fig. 1. Number of children with each goal and the rate of goal attainment.

\subsubsection{Goals to improve function and goal attainments}

Almost all children at GMFCS levels III to V used spinal orthoses to improve function with one or more of the following goals: improve stability/positioning; improve arm/hand function; or improve head control (Fig. 1). There were no significant differences in either goals or goal attainment levels in relation to age or sex (Table 2). Several goals were reported for most children. Improved stability and positioning were the most common treatment goals reported for 241 (96\%) of the 251 children, of whom 209 (87\%) attained the goal. These children ranged in age from 2 to 14 years. A higher proportion of children classified at GMFCS levels IV (99\%) and V (95\%) used spinal orthoses for stability/positioning ( $p=0.043$ ) compared with children classified at level III (75\%). Improved arm/hand function was a treatment goal for 96 children (38\%), of whom $77(80 \%)$ attained the goal. A significantly higher proportion of children classified at GMFCS level IV (51 of 77) used a spinal orthosis to improve arm/hand function $(p<0.001)$ compared with children at levels III (1 of 4) and V (44 of 170). Almost half of the 251 children $(n=127)$ used a spinal orthosis to improve head control, and $99(78 \%)$ of them attained this goal. Slightly more children with severe scoliosis used a spinal orthosis to improve head control; however, no clear association was found between the sever- 
ity of the scoliosis and goal attainment for any of the three functional goals (Table 2).

\subsubsection{Goal to prevent deformity and goal attainment}

Only one third of the children (83 of 251) used spinal orthoses to prevent deformity. Of these 83 children, $47(57 \%)$ attained the goal, meaning their spine was either stabilized with no progression of spinal deformity or had improved over the previous year. These children ranged in age from 3 to 14 years. There were no significant differences in the goal to prevent deformity or goal attainment in relation to age or sex (Table 2). A higher proportion of children with moderate $(41 \%)$ and severe scoliosis $(63 \%)$ used spinal orthoses to prevent deformity compared with children with no $(23 \%)$ or mild $(25 \%)$ scoliosis only visible during forward bending. Goal attainment to prevent deformity was proportional to the severity of the scoliosis, ranging from $71 \%$ reported success in children with mild scoliosis to $37 \%$ in children with severe scoliosis. A higher proportion of children at GMFCS level V (59 of 170) than at GMFCS level IV (23 of 77) used a spinal orthosis to prevent deformity. Furthermore, goal attainment was slightly higher for children classified at GMFCS level IV (61\%) than for those at GMFCS level V $(56 \%)$ (Table 2).

\subsection{Scoliosis}

The majority of children with scoliosis $(339,70 \%)$ were not treated with spinal orthoses. Scoliosis occurred as frequently in boys $(17 \%)$ as in girls $(17 \%)$. Nevertheless, a higher proportion of girls $(75,6.4 \%)$ than boys $(60,3.7 \%)$ had moderate or severe scoliosis $(p=0.002)$. The same difference was seen for children who underwent surgery, as almost twice as many girls $(26,2.2 \%)$ as boys $(16,1 \%)$ had a spinal fusion. Of the 486 children with scoliosis, the curvature was rated as mild in $333(69 \%)$, moderate in $71(15 \%)$, and severe in $64(13 \%)$, with missing data for 18 children. Scoliosis increased with age $(p<0.001)$ and was more frequent in children at lower levels of motor function ( $p<0.001)$, increasing from $92(7.6 \%)$ of the children at GMFCS level I to 198 (45\%) of the children at GMFCS level V. Of the children at GMFCS level I, only four $(0.3 \%)$ had moderate scoliosis, while $27(6.4 \%)$ at GMFCS level IV and 97 (22\%) at GMFCS level V had moderate or severe scoliosis.

\section{Discussion}

To our knowledge, this is the first study describing the use of spinal orthoses in a total population of chil- dren with CP. Slightly fewer than one in 10 children with CP used spinal orthoses. They were equally common in boys and girls, and usage increased with age and GMFCS level. Spinal orthoses were mostly used to improve functional outcomes, and the overall rate of goal attainment was high.

A stable posture and head control are important for optimizing function, although this can be challenging for children with severe postural deficits. For the vast majority $(96 \%)$, the primary goal for the orthosis was improvement in stability/positioning. Almost nine of 10 attained this goal, consistent with the $90 \%$ success rate reported by Letts et al. [10]. Improved sitting ability has previously been reported for individuals at GMFCS levels III-V who used soft, rigid, or dynamic spinal orthoses [24,25]. Not surprisingly, this treatment goal was reported more frequently for children at GMFCS levels IV and V than for children at level III, who usually require less trunk support. Improved head control was the second most common goal, and this was achieved in eight of 10 children, similar to the $85 \%$ success reported by Letts et al. [10]. Head control is also improved through better trunk stability $[7,8]$. Use of a spinal orthosis to improve head control was slightly more frequent in children with severe scoliosis than in other groups. Other abilities, such as communication and eating, are closely connected to head control [2]. Lack of stability seriously affects arm/hand function and leads to reduced wheelchair mobility [3], whereas improved arm/hand function can enhance play and independence in activities of daily living [7,8]. Twice as many children classified at GMFCS level IV than at levels III and V used a spinal orthosis to improve arm/hand function, highlighting the relationship between stability and arm/hand function for this group of children.

Only one in three of the children used a spinal orthosis to prevent or correct spinal deformity, making it the least common treatment goal. Goal attainment was reported by slightly fewer than three in five. These numbers are consistent with the results of a study by Olafsson et al. [12]. Seven in 10 children with mild scoliosis, but fewer than four in 10 with severe scoliosis, attained the goal of preventing spinal deformity, which indicated that the rate of goal attainment was proportional to the severity of the curvature. This may be explained by the better outcomes reported for spinal orthoses when used for smaller curvatures with radiographic Cobb angles $\leqslant 40^{\circ}[8,12]$. This suggests that the focus should be on preventive and early treatment rather than on efforts to stabilize a collapsing spine 
at a later stage [12,24]. A significantly higher proportion of girls than boys had moderate or severe spinal curvature, even though the overall prevalence was the same. Furthermore, almost twice as many girls underwent spinal fusion. This is consistent with the results of other studies that reported a slightly higher risk for scoliosis in girls $[15,26]$.

There are several limitations to this study. The goals for treatment and goal attainment levels were based on the performance in everyday life and not in a clinical setting, reported by the child's physiotherapist, the child, and the caregivers. This means that there was a subjective component to the assessment; however, this demonstrated how goal attainment was perceived by the families in various settings and conditions. The number of children wearing spinal orthoses differed between GMFCS levels, with only a small number at GMFCS level III wearing spinal orthoses. The study also lacked detailed information regarding the type of spinal orthoses, including their materials and whether they were prefabricated or individually molded. In Sweden, the public health care system generally functions well, with free health care, orthotics, and assistive devices available for children with disabilities. There is no international consensus on the use of spinal orthoses; therefore, their use is likely to vary between countries. This was a cross-sectional study, where the design was used to document the status of a group at a particular point in time. It does not reflect changes over time. Nevertheless, a strength of this study was that all data had been systematically reported for the total population of children with $\mathrm{CP}$ in Sweden at all GMFCS levels, CP subtypes, and in both rural and urban settings.

\section{Conclusions}

Most children who use a spinal orthosis do so to improve function rather than prevent deformity. Goal attainment levels were high for stability, head control, and arm/hand function, indicating functional benefits for children in their daily lives. There appear to be higher goal attainment levels for prevention of deformity when spinal orthoses are used for mild scoliosis, and lower goal attainment levels when they are used for moderate or severe scoliosis. Because the ultimate treatment goal is to improve activity, participation, and quality of life, children with postural deficits should be given the opportunity to explore the functional benefits of a spinal orthosis.

\section{Acknowledgments}

We would like to thank the Norrbacka-Eugenia Foundation for grants and Stiftelsen för bistånd åt rörelsehindrade i Skåne for financial support.

\section{Conflict of interest}

The authors have no conflict of interest to report.

\section{References}

[1] Rodby-Bousquet E, Czuba T, Hägglund G, Westbom L. Postural asymmetries in young adults with cerebral palsy. Dev Med Child Neurol. 2013; 55: 1009-1015. doi: 10.1111/dmen. 12199.

[2] Carlberg EB, Hadders-Algra M. Postural dysfunction in children with cerebral palsy: Some implications for therapeutic guidance. Neural Plast. 2005; 12: 221-228; discussion 263272. doi: 10.1155/NP.2005.221.

[3] Lacoste M, Therrien M, Prince F. Stability of children with cerebral palsy in their wheelchair seating: Perceptions of parents and therapists. Disabil Rehabil Assist Technol. 2009; 4 143-150. doi: S10.1080/17483100802362036.

[4] Saito N, Ebara S, Ohotsuka K, Kumeta H, Takaoka K. Natural history of scoliosis in spastic cerebral palsy. Lancet. 1998; 351: 1687-1692. doi: 10.1016/S0140-6736(98)01302-6.

[5] Palisano RJ, Rosenbaum P, Bartlett D, Livingston MH. Content validity of the expanded and revised gross motor function classification system. Dev Med Child Neurol. 2008; 50 : 744-750. doi: 10.1111/j.1469-8749.2008.03089.x.

[6] Morrell DS, Pearson JM, Sauser DD. Progressive bone and joint abnormalities of the spine and lower extremities in cerebral palsy. Radiographics. 2002; 22: 257-268. doi: 10.1148/ radiographics.22.2.g02mr19257.

[7] Rutz E, Brunner R. Management of spinal deformity in cerebral palsy: Conservative treatment. J Child Orthop. 2013; 7 : 415-418. doi: 10.1007/s11832-013-0516-5.

[8] Terjesen T, Lange JE, Steen H. Treatment of scoliosis with spinal bracing in quadriplegic cerebral palsy. Dev Med Child Neurol. 2000; 42: 448-454.

[9] Miller A, Temple T, Miller F. Impact of orthoses on the rate of scoliosis progression in children with cerebral palsy. J Pediatr Orthop. 1996; 16: 332-335. doi: 10.1097/01241398199605000-00007.

[10] Letts M, Rathbone D, Yamashita T, Nichol B, Keeler A. Soft Boston orthosis in management of neuromuscular scoliosis: A preliminary report. J Pediatr Orthop. 1992; 12: 470-474.

[11] Nachemson AL, Peterson LE. Effectiveness of treatment with a brace in girls who have adolescent idiopathic scoliosis. A prospective, controlled study based on data from the brace study of the scoliosis research society. J Bone Joint Surg Am. 1995; 77: 815-822.

[12] Olafsson Y, Saraste H, Al-Dabbagh Z. Brace treatment in neuromuscular spine deformity. J Pediatr Orthop. 1999; 19: 376-379.

[13] Imrie MN, Yaszay B. Management of spinal deformity in cerebral palsy. Orthop Clin North Am. 2010; 41: 531-547. doi: 10.1016/j.ocl.2010.06.008. 
[14] Koop SE. Scoliosis in cerebral palsy. Dev Med Child Neurol. 2009; 51: 92-98. doi: 10.1111/j.1469-8749.2009.03461.x.

[15] Hägglund G, Pettersson K, Czuba T, Persson-Bunke M, Rodby-Bousquet E. Incidence of scoliosis in cerebral palsy. Acta Orthop. 2018; 1-5. doi: 10.1080/17453674.2018.14500 91.

[16] Kotwicki T, Jozwiak M. Conservative management of neuromuscular scoliosis: Personal experience and review of literature. Disabil Rehabil. 2008; 30: 792-798. doi: 10.1080/ 09638280801889584.

[17] Tsirikos AI, Spielmann P. Spinal deformity in paediatric patients with cerebral palsy. Current Orthopaedics. 2007; 21: 122-134. doi: 10.1016/j.cuor.2007.01.001.

[18] Hägglund $G$, Alriksson-Schmidt A, Lauge-Pedersen $H$, Rodby-Bousquet E, Wagner P, Westbom L. Prevention of dislocation of the hip in children with cerebral palsy: 20-year results of a population-based prevention programme. Bone Joint J. 2014; 96-B: 1546-1552. doi: 10.1302/0301-620X.96B11. 34385 .

[19] Hägglund G, Andersson S, Duppe H, Lauge-Pedersen H, Nordmark E, Westbom L. Prevention of dislocation of the hip in children with cerebral palsy. The First Ten Years of a Population-based Prevention Programme. J Bone Joint Surg Br. 2005; 87: 95-101.

[20] Westbom L, Hagglund G, Nordmark E. Cerebral palsy in a total population of 4-11 year olds in southern sweden. Prevalence and Distribution According to Different CP Classification Systems. BMC Pediatr. 2007; 7: 41. doi: 10.1186/14712431-7-41.
[21] S.o.C.P.i.E. (SCPE), Surveillance of cerebral palsy in Europe: a collaboration of cerebral palsy surveys and registers. Dev Med Child Neurol. 2000; 42: 816-824.

[22] Alriksson-Schmidt AI, Arner M, Westbom L, KrumlindeSundholm L, Nordmark E, Rodby-Bousquet E, et al. A combined surveillance program and quality register improves management of childhood disability. Disabil Rehabil. 2017; 39: 830-836. doi: 10.3109/09638288.2016.1161843.

[23] Persson-Bunke M, Czuba T, Hägglund G, Rodby-Bousquet E. Psychometric evaluation of spinal assessment methods to screen for scoliosis in children and adolescents with cerebral palsy. BMC Musculoskelet Disord. 2015; 16: 351. doi: 10.1186/s12891-015-0801-1.

[24] Nakamura N, Uesugi M, Inaba Y, Machida J, Okuzumi S, Saito T. Use of dynamic spinal brace in the management of neuromuscular scoliosis: A preliminary report. J Pediatr Orthop B. 2014; 23: 291-298. doi: 10.1097/bpb.000000000000 0034 .

[25] Leopando MT, Moussavi Z, Holbrow J, Chernick V, Pasterkamp H, Rempel G. Effect of a soft boston orthosis on pulmonary mechanics in severe cerebral palsy. Pediatr Pulmonol. 1999; 28: 53-58.

[26] Bertoncelli CM, Solla F, Loughenbury PR, Tsirikos AI, Bertoncelli D, Rampal V. Risk factors for developing scoliosis in cerebral palsy: A cross-sectional descriptive study. J Child Neurol. 2017; 32: 657-662. doi: 10.1177/0883073817 701047. 